\title{
A Comparative Study on the Chest CT Scan Radiological Findings and Hematological Parameters of COVID-19 and NON-COVID-19 Pneumoniapatients in Al-Yarmouk Teaching Hospital in Baghdad, Iraq
}

\author{
Bassam Maddah Hassan ${ }^{1}$, Mothana Ali Khalil'², \\ Rana Adnan Abdulhameed ${ }^{3}$, Abbas Jaafar Khaleel Al-Anbari ${ }^{4}$ \\ ${ }^{1}$ Surgical Department, Lecturer, MBCHB, FIBMS/Anbar Medical College/University of Anbar/Iraq, ${ }^{2}$ Assistant \\ Professor/Anbar Medical College, Medical Microbiology Department,/University of Anbar/Iraq, ${ }^{3}$ Specialist \\ Diagnostic Radiology, Al Yarmouk Teaching Hospital Baghdad/MOH/Iraq, ${ }^{4}$ MBCHB, FIBMS Al-Nahrain \\ University, College of Medicine, Surgical Department/Iraq
}

\begin{abstract}
Background: Chest CT scan has a great sensitivity with rapid diagnoses of COVID-19 infection in the community. Nevertheless, the specificity of this test for diagnosis of this disease are believed to be low.

Objective: To evaluate role, effectiveness and diagnostic accuracy of chest CT scan with main imaging manifestations and hematological appearances for screening COVID-19 cases through comparing results of those with other NON-COVID-19 pneumonia.

Patients and Method: Twenty COVID-19 \& 28NON-COVID-19pneumoniaIraqi patients in Yarmouk teaching hospital, Baghdad, Iraq from 1 march to 1 may 2020, at the beginning of the outbreak in Iraq COVID-19 had been included in current research. Confirmed COVID-19 together with NON-COVID-19 patients to be infected or not with SARS-CoV-2 using RT-PCR were included in current research. Sociodemographic,hematological and chest CT scan finding were examined to compare the variance between COVID-19 and NON-COVID-19 patients.
\end{abstract}

Result: "COVID-19" patients mean age was $62.10 \pm 14.308$ SDrangedbetween 35- 90 years whereas "non-COVID-19" patients mean was 61.07 \pm 13.638 SD. There was a significant difference between WBC average number of COVID-19 compared to its average number of "NON-COVID-19" patients $(\mathrm{P}<0.001)$. A significant differences between Neutrophils, lymphocytes, Monocytes, Eosinophil and Basophils low counts and MCV, MCH, PDW of COVID-19 compared to its counts or levels in "NON-COVID-19" patients $(\mathrm{P}<0.001)$. A significantly different of CT scanImages 'Ground glass opacity, Crazy paving alteration', 'Vascular dilatations, Traction Bronchiectasis alteration', 'Sub pleural -bands' \& Architectura) between "COVID-19" \& "NON-COVID-19 cases (P less than 0.001), whereas Consolidation was non-significant.

Conclusion: A chest CT scan might be a dependabletool for COVID-19 cases diagnosis. Hematological features that include WBC, Neutrophils, Lymphocytes, Monocytes, Eosinophil, Basophils, MCV, MCH, PDW might be substantial indications for COVID-19 appraisal.

Keywords: COVID-19, NON-COVID-19, Pneumonia, CT scan, Hematological test,case-control study.

\section{Corresponding Author:}

Mothana Ali Khalil

Assistant Professor/Anbar Medical College, Medical Microbiology Department,/University of Anbar/Iraq e-mail: mothanakhalil70@yahoo.com

\section{Introduction}

〈Coronavirus〉 interactive 〈disease-2019〉 (COVID-19) is a pandemic outbreakproduced by 〈severe acute respiratory syndrome〉 coronavirus-2 (SARS-CoV-2. It begins suddenly in December 2019 
in 'Wuhan' City, 'China', then spread in 213 countries characterized by a minor common cold-like sickness, to 'severe viral- pneumonia' might develop to' acuterespiratory-distress-syndrome' that is possiblylethal)1).

RT-PCR sensitivity might be lower than 50\% \& certain diagnoses might be lost.,Low sensitivityIn 'COVID 19' early stage, the results of this test may take more than 24 hours, while the results of a CT scan are available immediately ${ }^{2)}$. RT-PCR test for SARS-CoV-2 viral RNA recognition is very specific but low sensitive ${ }^{\text {(3) }}$ as greatest of COVID-19 cases with duplication negative results. Chest CT scan lung findings are appearthan clinical manifestations, which make Chest CT scan critical for runs and accurate diagnosis ${ }^{4)}$. So,CT scan might be applied as an supplementary diagnostic tool simultaneously in COVID-19 diagnosis) ${ }^{2}$.

Chest CT scans has high sensitivity with low specificity in COVID-19, so it is problematic to differentiate COVID-19 from non-COVID-19 pneumonia cases $)^{5,6)}$. Nevertheless, RT-PCR appear as negative results, while their $\mathrm{CT}$ imaging findings were abnormal as reported in certain study) $)^{7,8,9,10)}$ Through using of chest CT scan findings with RT-PCR, the sensitivity could be reach to $99 \%$, so chest CT scan should be used as a reference for COVID-19 dignosis ${ }^{6}$.

Chinese studies related to COVID-19 showed that there is a decrease in white blood cells in hospital patients, with varying degrees of decrease in lymphocytes with a relative decrease in platelets ${ }^{11)}$.

In Iraq, as far as we know, no previous study has yet compared chest CT scan and hematological investigation results between COVID-19 \& nonCOVID-19 viral pneumonia kinds. Thus, this study was the first research to compare chest CT scan findings \& hematological features of COVID-19 \& non-COVID19viral pneumonia kindsto know diagnostic efficacy of these tools inCOVID-19 diagnosis.

\section{Patients and Method}

Prospective case-control design was used to identify the role, effectiveness and diagnostic accuracy of hematological tests with a chest CT scanfor screening and rapid diagnosis ofCOVID-19 infections through compared the difference of Hematological tests and radiographic features results of "COVID-19" \& "NONCOVID-19" viral pneumonia kinds. Twenty "COVID 19 "together with pneumonia patients 28 other" NON-
NCOVID-19" were confirmed simultaneously to be infected or not infected with SARS-CoV-2 respectively using RT-PCR \& blood as a suitable specimen in Yarmouk teaching hospital, Baghdad, Iraq from 1 march to 31 April 2020, at the beginning of the outbreak in Iraq COVID-19. We analyzed the demographic, compare the pulmonary CT scan findings and hematological investigation among "COVID-19" \& NON-COVID-19 Iraqi patients. Anbar medical College approved the protocol. All patients provided informed consent for participation in the study.

Statistical Analyses: Data were analyzed using IBM SPSS software version 22. The results were presented in tables as frequencies and percentages. ChiSquare test was used to compare between the variables through cross-tabulations and P-Value less than 0.05 considered a statistically significant difference

\section{Results}

Twenty COVID-19 confirmed caseswith 28 chest pain non-COVID-19 cases (as a control) were enrolled in the study.

'COVID-19' patients mean age was 62.10 \pm 14.308 SD ranged between 35 months and 90years whereas 'non COVID-19' patients mean age was 61.07士 13.638 SD, Age group had been categorized as following: $<=43(2,10.0 \%) ; 44-59(5,25 \%) ; 60-74(9,45.0 \%)$ and $75+$ years $(4,20.0 \%),(11,55.0 \%)$ and $(9,45.0 \%)$ were male and female respectively .Fifteen (75.0\%)of COVID-19 patients were identified during March and others 5 (25.0\%) identified during April 2020.

Table 1: Epidemiological characteristics of COVID-19 confirmed Patient diagnosed in AlYarmouk teaching hospital, Baghdad, Iraq

\begin{tabular}{|l|c|}
\hline Character & Frequency (\%) (N. 20) \\
\hline Mean of age & $62.10 \pm 14.308$ SD \\
\hline Gender & $11(55.0 \%)$ \\
\hline Male & $9(45.0 \%)$ \\
Female & $15(75.0 \%)$ \\
\hline Incidence in month & $5(25.0 \%)$ \\
\hline March \\
April & \\
\hline Age group & $2(10.0 \%)$ \\
\hline$<=43$ & $5(25 \%)$ \\
$44-59$ & $9(45.0 \%)$ \\
$60-74$ & $420.0 \%$ \\
$75+$
\end{tabular}


Normal WBC average number $(6.480 / \mathrm{mcL}$ in COVID-19 (20 [41.7\%]) compare with elevation of WBC average number $(10.193 / \mathrm{mcL})(28$ [58.3\%]) of NON-COVID-19 patients, significant difference of WBC average number of COVID-19 compare withNONCOVID-19 cases $(\mathrm{P}<0.001)$.

Lymphocytes were decreased in COVID-19(20[41.7\%]) compare with NON-COVID-19 patients $(\mathrm{P}<0.001)(28[58.3 \% \%]$.

Decreased count or levels of Neutrophils, lymphocyt es,Monocytes,Eosinophil and Basophilswas observed in COVID-19 (4.500/mcL), (1.330/mcL) (0.350), (0.075) (0.035) respectively Compared to $(6.679 / \mathrm{mcL}),(2.154 /$ $\mathrm{mcL}),(0.782)(0.429), 0.150)$ respectively of NONNCOVID-19 patients. A significant difference among Neutrophils, lymphocytes, Monocytes, Eosinophil and Basophils low levels of COVID-19 Compared to its counts in NON -COVID-19 patients $(\mathrm{P}<0.001)$.

Abnormally decreased Mean Corpuscular Volume
(MCV) and corpuscular Hemoglobin (MCH)were seen in COVID-19 patients (85.360fL), (28.520pg) respectively Compared to its Mean (89.061fL), (29.939pg) respectively in NON-NCOVID-19 patients. A significant difference among decrease mean MCVand MCH of COVID-19 compared to its Mean in NONCOVID-19 patients $(\mathrm{P}<0.001)$ (Table 3$)$.

Abnormally increased Platelet Distribution Width (PDW) was seen in COVID-19 patients (17.762\%) compared to its percent (15.896\%) in 'non-COVID-19' patients, a significant difference among increase PDW of COVID-19 Compared to its percent in NON-COVID-19 patients $(\mathrm{P}<0.001)$ (Table 3).

Whoever, non-significant difference amongst level of RBCs (P. Value 0.474), 'hemoglobin' (P. Value 0.135), 'Hematocrit-test' (P. Value 0.060), 'Platelets Count'(P. Value 0.121), 'mean platelet volume' (P. Value 0.710) \& PCT levels (P. Value 0.372 ) of 'COVID-19' \& 'nonCOVID-19' patients.

Table 2: Statistical comparisons different among' COVID-19' \& NON-COVID-19 (as Control) hematological parameters

\begin{tabular}{|c|c|c|c|c|c|c|}
\hline Parameter & $\begin{array}{l}\text { Confirmed COVID-19, NON- } \\
\text { COVID-19 cases (Total No.48) }\end{array}$ & $\mathbf{N}$ & Mean & Std. Deviation & Std. Error Mean & P-value \\
\hline \multirow{2}{*}{ WBCcount } & COVID-19 & 20 & 6.480 & 2.1910 & 0.4899 & \multirow{2}{*}{0.000} \\
\hline & Non-COVID-19 & 28 & 10.193 & 3.8400 & 0.7257 & \\
\hline \multirow{2}{*}{ Neutrophils } & COVID-19 & 20 & 4.500 & 2.0532 & 0.4591 & \multirow{2}{*}{0.002} \\
\hline & Non-COVID-19 & 28 & 6.679 & 2.4524 & 0.4635 & \\
\hline \multirow{2}{*}{ Lymphocytes } & COVID-19 & 20 & 1.330 & 0.4566 & 0.1021 & \multirow{2}{*}{0.002} \\
\hline & Non-COVID-19 & 28 & 2.154 & 1.0500 & 0.1984 & \\
\hline \multirow{2}{*}{ Monocytes } & COVID-19 & 20 & 0.350 & 0.0889 & 0.0199 & \multirow{2}{*}{0.000} \\
\hline & Non-COVID-19 & 28 & 0.782 & 0.2653 & 0.0501 & \\
\hline \multirow{2}{*}{ Eosinophil } & COVID-19 & 20 & 0.075 & 0.0786 & 0.0176 & \multirow{2}{*}{0.000} \\
\hline & Non-COVID-19 & 28 & 0.429 & 0.3516 & 0.0664 & \\
\hline \multirow{2}{*}{ Basophils } & COVID-19 & 20 & 0.035 & 0.0489 & 0.0109 & \multirow{2}{*}{0.008} \\
\hline & Non-COVID-19 & 28 & 0.150 & 0.1816 & 0.0343 & \\
\hline \multirow{2}{*}{ RBC level } & COVID-19 & 20 & 4.6215 & 0.78433 & 0.17538 & \multirow{2}{*}{0.474} \\
\hline & Non-COVID-19 & 28 & 4.7571 & 0.51742 & 0.09778 & \\
\hline \multirow{2}{*}{$\mathrm{HB}$} & COVID-19 & 20 & 12.640 & 1.9749 & 0.4416 & \multirow{2}{*}{0.135} \\
\hline & Non-COVID-19 & 28 & 11.843 & 1.6428 & 0.3105 & \\
\hline \multirow{2}{*}{$\begin{array}{l}\text { Hematocrit } \\
\text { test }\end{array}$} & COVID-19 & 20 & 39.605 & 6.1581 & 1.3770 & \multirow{2}{*}{0.060} \\
\hline & Non-COVID-19 & 28 & 42.946 & 5.7403 & 1.0848 & \\
\hline
\end{tabular}




\begin{tabular}{|c|c|c|c|c|c|c|}
\hline Parameter & $\begin{array}{l}\text { Confirmed COVID-19, NON- } \\
\text { COVID-19 cases (Total No.48) }\end{array}$ & $\mathbf{N}$ & Mean & Std. Deviation & Std. Error Mean & P-value \\
\hline \multirow{2}{*}{$\mathrm{MCV}$} & COVID-19 & 20 & 85.360 & 4.0512 & 0.9059 & \multirow{2}{*}{0.024} \\
\hline & Non-COVID-19 & 28 & 89.061 & 6.1818 & 1.1683 & \\
\hline \multirow{2}{*}{$\mathrm{MCH}$} & COVID-19 & 20 & 28.520 & 2.0943 & 0.4683 & \multirow{2}{*}{0.020} \\
\hline & Non-COVID-19 & 28 & 29.939 & 1.9547 & 0.3694 & \\
\hline \multirow{2}{*}{ Platelet Count } & COVID-19 & 20 & 188.80 & 53.211 & 11.898 & \multirow{2}{*}{0.121} \\
\hline & Non-COVID-19 & 28 & 217.11 & 66.143 & 12.500 & \\
\hline \multirow{2}{*}{ PCT } & COVID--19 & 20 & .1315 & 0.03183 & 0.00712 & \multirow{2}{*}{0.372} \\
\hline & Non-COVID-19 & 28 & .1243 & 0.02364 & 0.00447 & \\
\hline \multirow{2}{*}{$\begin{array}{l}\text { Mean platelet } \\
\text { volume }\end{array}$} & COVID-19 & 20 & 7.429 & 0.6587 & 0.1473 & \multirow{2}{*}{0.710} \\
\hline & Non-COVID-19 & 28 & 7.539 & 1.1970 & 0.2262 & \\
\hline \multirow{2}{*}{ PDW } & COVID-19 & 20 & 17.762 & 0.8898 & 0.1990 & \multirow{2}{*}{0.000} \\
\hline & Non-COVID-19 & 28 & 15.896 & 0.5189 & 0.0981 & \\
\hline
\end{tabular}

A Chest CT scan of COVID-19 patients with NONCOVID-19 patients had been shown in Figure 1,2,3 and Table 3.

Ground glass was present in $17 / 20$ patients $(85.0 \%)$ in COVID-19 confirmed cases, in 9/28 patients (32.1\%) in NON-COVID-19 cases. Crazy paving was present in $15 / 20$ patients $(75.0 \%)$ in COVID-19 confirmed cases and only in $2 / 28$ patients $(7.1 \%)$ in Chest pain Unconfirmed COVID-19 cases. A significant difference was observed between the COVID-19 confirmed cases and Chest pain NON- COVID-19 cases regarding the ground glass and Crazy paving alteration ( $\mathrm{P}$ Value= 0.000) Table 3.

Vascular dilatation was present in $12 / 20$ patients (60.0\%) in COVID-19 confirmed cases and only in $2 / 28$ patients $(7.1 \%)$ in Chest pain unconfirmed COVID-19 cases. Traction Bronchiectasis was present in 11/20 patients (55.0\%) in COVID-19 confirmed cases and only in $3 / 28$ patients $(10.7 \%)$ in Chest pain unconfirmed COVID-19 cases. Subpleural bands and Architectural were present in 10/20 patients (50.0\%) in COVID-19 confirmed cases and only in $3 / 28$ patients $(10.7 \%)$ in
Chest pain unconfirmed COVID-19 cases Significant difference was observed between the COVID-19 confirmed cases and Chest pain unconfirmed COVID-19 cases regarding the Vascular dilatation, Traction Bronchiectasisalteration and Subpleural bands and Architectural (P Value $=0.000)$ Table 3.

Consolidation was present only in $4 / 20$ patients (20.0\%) in COVID-19 confirmed cases and only in $15 / 28$ patients $(53.6 \%)$ in Chest pain unconfirmed COVID-19 cases, non- significant difference had been observed between the COVID-19 confirmed cases and Chest pain unconfirmed COVID-19 cases regarding the Consolidation alteration $(\mathrm{P}$ Value $=0.035)$ Table 3.

Ground -glass opacity, Crazy- paving alteration, wide Vascular-- dilatation, Traction-Bronchiectasis alteration, Sub - pleural-bands \& Architecture finding of Chest CT the were significantly associated with COVID-19 pneumonia, whereas Consolidation was not significantly associated with COVID-19 pneumonia $(\mathrm{P}<0.001)$, so chest CT scancan be documented as a preliminary diagnosic tool of COVID . 


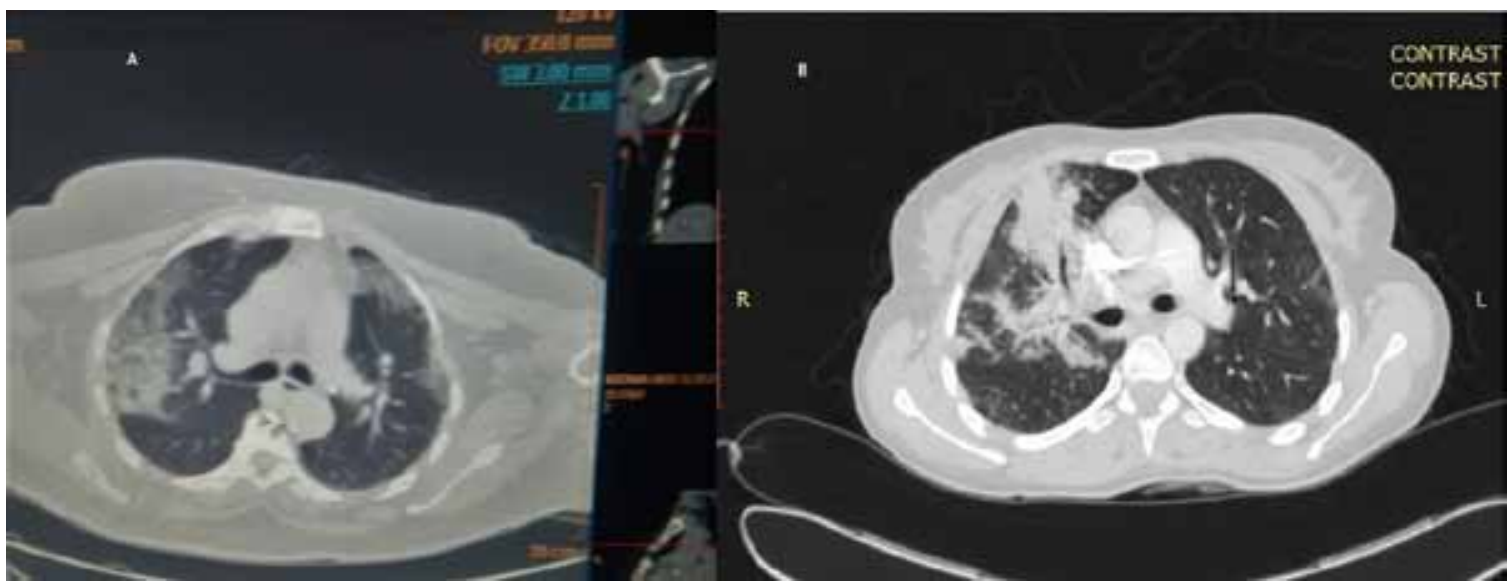

Figure 1: A. COVID-19 positive CT picture of the crazy paving pattern, B. Segmental consolidation in a patient with severe chest infection COVID-19 negative patient

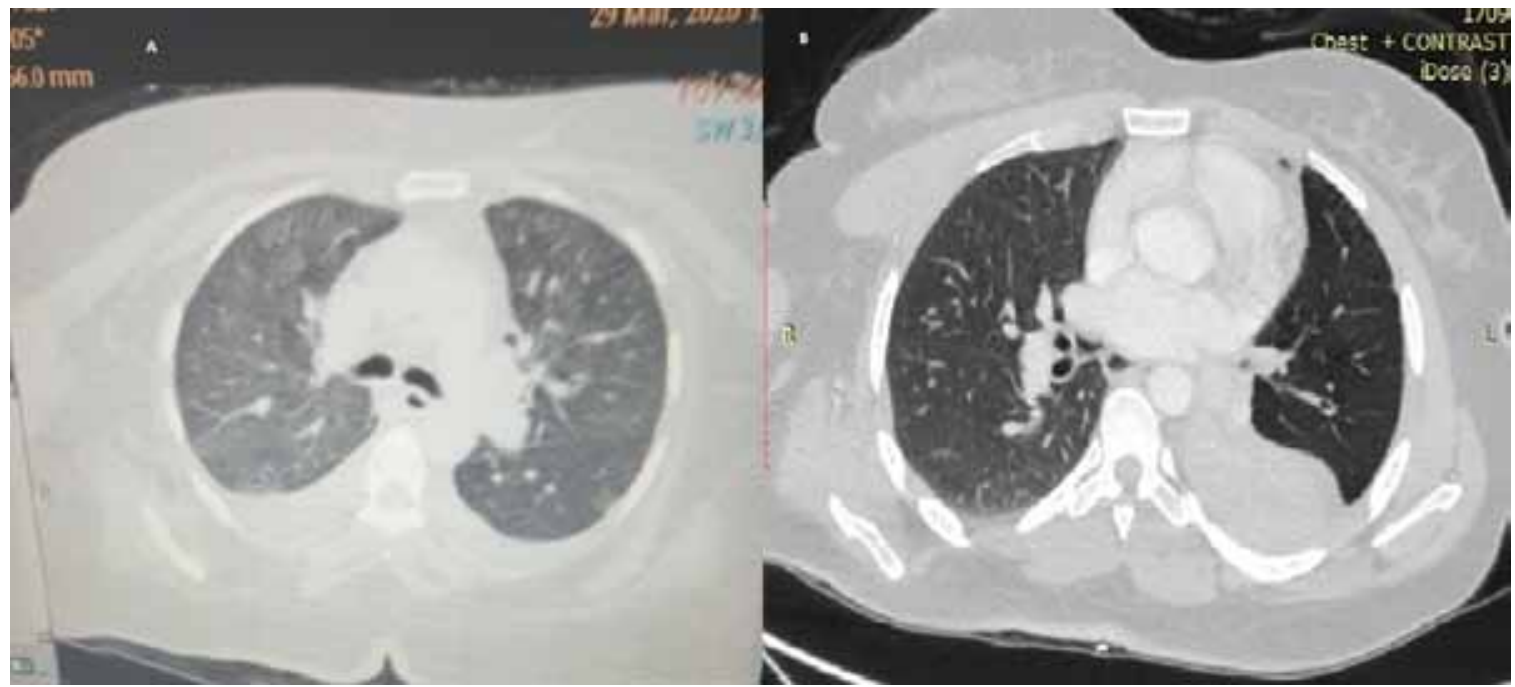

Figure 2: A. Typical diffuse ground glass appearance with bilateral pleural effusion in COVID-19 positive patient, B. Nodular ground-glass pneumonitis with left pleural effusion COVID-19 negative patient.
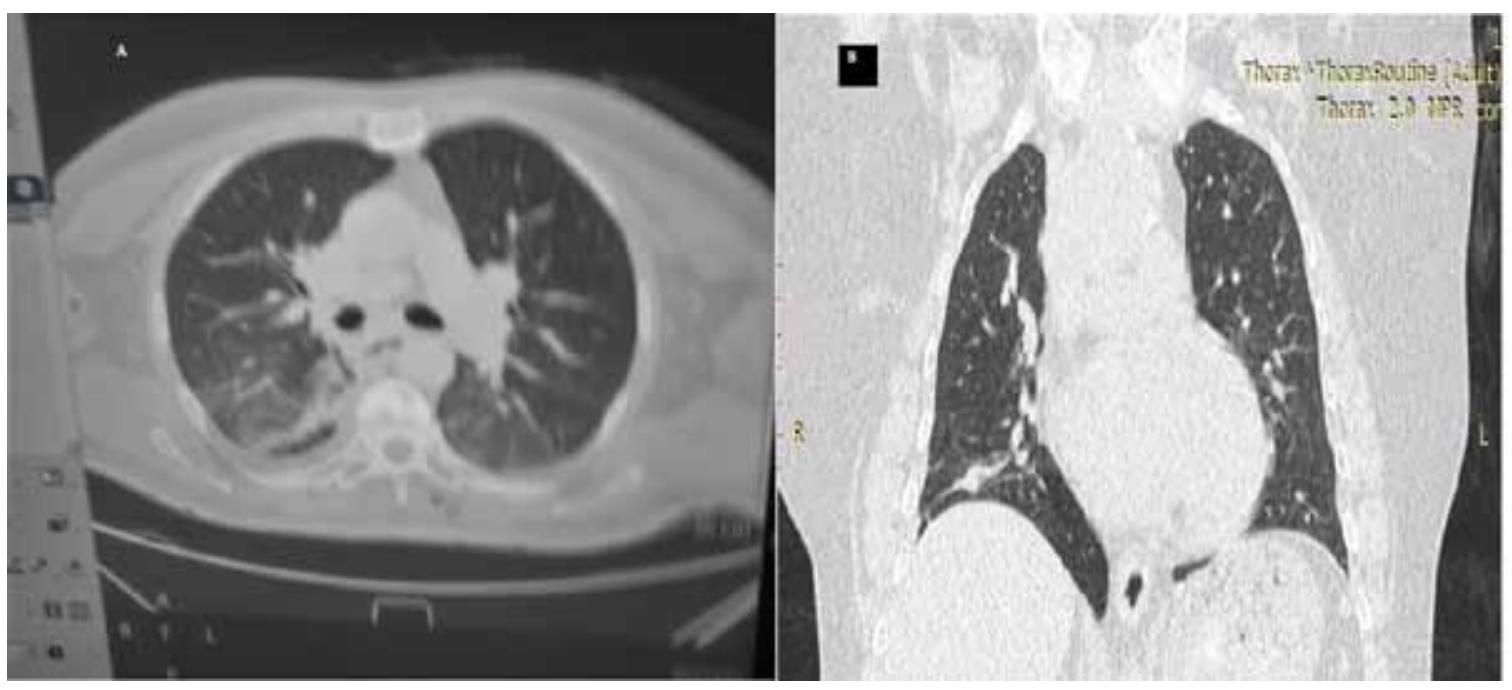

Figure 3: A. Typical septal thickening with ground glass appearance in COVID-19 positive patient, B. Septal thickening and tree in bud pattern of trans-bronchial spread COVID-19 negative patient. 
Table 3: Statistical comparisons of different chest CT scan Radiological diagnostic features,lesions and imaging manifestations among COVID-19 confirmed cases \& NON-COVID-19 (Control).

\begin{tabular}{|c|c|c|c|}
\hline Character & $\begin{array}{l}\text { COVID-19 confirmed cases } \\
(\mathrm{N}=20), \text { frequency }(\%)\end{array}$ & $\begin{array}{l}\text { NON-COVID-19 cases (Control) } \\
(\mathrm{N}=28), \text { frequency }(\%)\end{array}$ & P. value \\
\hline \multicolumn{4}{|l|}{ Ground glass } \\
\hline Positive feature & $17(85.0 \%)$ & $9(32.1 \%)$ & \multirow{2}{*}{0.000} \\
\hline Negative feature & $3(15.0 \%)$ & $19(67.9 \%)$ & \\
\hline \multicolumn{4}{|l|}{ Crazy paving } \\
\hline Positive feature & $15(75.0 \%)$ & $2(7.1 \%)$ & \multirow{2}{*}{0.000} \\
\hline Negative feature & $5(25.0 \%)$ & $26(92.9 \%)$ & \\
\hline \multicolumn{4}{|c|}{ Vascular dilatation } \\
\hline Positive feature & $12(60.0 \%)$ & $2(7.1 \%)$ & \multirow{2}{*}{0.000} \\
\hline Negative feature & $8(40.0 \%)$ & $26(92.9 \%)$ & \\
\hline \multicolumn{4}{|c|}{ Traction Bronchiectasis } \\
\hline Positive feature & $11(55.0 \%)$ & $3(10.7 \%)$ & \multirow{2}{*}{0.001} \\
\hline Negative feature & $9(45.0 \%)$ & $25(89.3 \%)$ & \\
\hline \multicolumn{4}{|c|}{ Sub pleural bands and Architectural } \\
\hline Positive feature & $10(50.0 \%)$ & $3(10.7 \%)$ & \multirow{2}{*}{0.003} \\
\hline Negative feature & $10(50.0 \%)$ & $25(89.3 \%)$ & \\
\hline \multicolumn{4}{|l|}{ Consolidation } \\
\hline Positive feature & $4(20.0 \%)$ & $15(53.6 \%)$ & \multirow{2}{*}{0.035} \\
\hline Negative feature & $16(80.0 \%)$ & $13(46.4 \%)$ & \\
\hline
\end{tabular}

\section{Discussion}

The current research aimed to show chest CT scan findings variance among COVID-19 \& non-COVID-19 patienes .

Current study revealed that chest CT scans can distinguish between COVID -19 from non-COVID-19 patience with in elevation specificity with a moderate sensitivity. This results had been agreement with the result reported by Bai et al. 2020)12).

The current study indicated that $85.0 \%$ of COVD19 showed initial abnormal CT scan findings, which supports the possibility of CT scan as an assisting diagnostic tool in addition to RT- PCR for COVD19 diagnosis. Most common CT scan finding or imaging in our patients was ground- glass \& two-sided peripheral spreading. Current study had been similar to previous report that showed that ground -glass was a chiefchest CT scan findings in COVID-19) ${ }^{13)}$.

However, It is worth noting that a small number of COVID 19 patients in our current study did not have CT scan abnormal change and this is consistent with a previous study that showed that $11.5 \%$ of patients with confirmed infection with this disease showed normal chest $\mathrm{CT}$ scan finding ${ }^{13)}$, so we conclude that a CT scan 
finding alone is not sufficient to fully exclude reliable and confirmed diagnosis of COVID 19, especially in the early stage of the disease .

Recent study that showed consolidation imaging not characteristic for COVID-19 pneumonia, these result was disagreement with the results reported by Pan et al. ${ }^{6)} \&$ Xie et al. ${ }^{(7)}$ that exhibited imaging consolidation as a characteristics for COVID-19 pneumonia.

The current study were consistent with the findings of previous report that showed crazy-paving patterns \& diffuse- distribution had been characteristic for CT findings of COVID-19 pneumonia $^{14, \text {, }}$.

Results of current study also in agreement with the result reported by previous study that demonstrate chest CT scan imaging are frequently normal in initialdisease phases; nevertheless, it mightdemonstration bilateralinfiltrates \& ground-glass opacity in initial disease phases $^{15)}$.

Results of the hematological finding in preset study was in agreement with results of previous study the presented varying degrees of laboratory abnormalities e.g. leukopenia, leukocytosis, lymphopenia among COVID-19 \& non-COVID-19 such as leukopenia, leukocytosis \& lymphopenia .... etc ${ }^{15}$.

Laboratory findingsmight be applied as an supplementary diagnostic tool simultaneously with chest CT scan,itacts mostly on lymphocytes which decease during COVID-19 but it still normal in nonCOVID-19) $)^{11,16)}$.

We suggested that current research on CT scan findings and imagines have highlighted the chest CT scan role in pandemic COVID-19 diagnosis. Researches with big sample size \& clear chest CT scan findings or imagines from different countries are required to guide usage of this tool in the COVID-19 diagnosis.

Ethical Clearance: The Research Ethical Committee at scientific research by ethical approval of both MOH and MOHSER in Iraq.

Conflict of Interest: Non

Funding: Self-funding

\section{References}

1. Xu X-W, Wu X-X, Jiang X-G, Xu K-J, Ying L-J, Ma C-L, et al. Clinical findings in a group of patients infected with the 2019 novel coronavirus (SARSCov-2) outside of Wuhan, China: retrospective case series. bmj. 2020 Feb 19;368.

2. Zhang S, Li H, Huang S, You W, Sun H. Highresolution computed tomography features of 17 cases of coronavirus disease 2019 in Sichuan province, China. Eur Respir J. 2020 Apr 1;55 (4).

3. Tan W, Zhao X, Ma X, Wang W, Niu P, Xu W, et al. A novel coronavirus genome identified in a cluster of pneumonia cases - Wuhan, China 20192020. China CDC Wkly. 2020;2(4):61-2.

4. Xiong Y, Sun D, Liu Y, Fan Y, Zhao L, Li X, et al. Clinical and high-resolution CT features of the COVID-19 infection: comparison of the initial and follow-up changes. Invest Radiol. 2020; Volume 55, Number 6: 1-8

5. Fang Y, Zhang H, Xie J, Lin M, Ying L, Pang P, Ji W. Sensitivity of chest CT for COVID-19: comparison to RT-PCR. Radiology. 2020. Volume 296: Number 2: E115-E117

6. Pan F, Ye T, Sun P, Gui S, Liang B, Li L, et al. Time course of lung changes on chest $\mathrm{CT}$ during recovery from 2019 novel coronavirus (COVID-19) pneumonia. Radiology. 2020; 295:715-721.

7. Xie X, Zhong Z, Zhao W, Zheng C, Wang F, Liu J. Chest CT for typical 2019-nCoV pneumonia: relationship to negative RT-PCR testing. Radiology. 2020; 296:E41-E45.

8. Bernheim A, Mei X, Huang M, Yang Y, Fayad ZA, Zhang N, et al. Chest $\mathrm{CT}$ findings in coronavirus disease-19 (COVID-19): relationship to duration of infection. Radiology. 2020; 295:685-691.

9. Ai T, Yang Z, Hou H, Zhan C, Chen C, Lv W, et al. Correlation of chest CT and RT-PCR testing in coronavirus disease 2019 (COVID-19) in China: a report of 1014 cases. Radiology. 2020 Aug;296(2):E32-E40.

10. Fang Y, Zhang H, Xie J, Lin M, Ying L, Pang $\mathrm{P}$, et al. Sensitivity of chest CT for COVID-19: comparison to RT-PCR. Radiology. 020. Volume 296: Number 2: E115-E117.

11. Fan BE, Chong VCL, Chan SSW, Lim GH, Lim KGE, Tan GB, et al. Hematologic parameters in patients with COVID-19 infection. Am J Hematol. 2020;95(6):E131-4.

12. Bai HX, Hsieh B, Xiong Z, Halsey K, Choi JW, Tran TML, et al. Performance of radiologists in differentiating COVID-19 from viral pneumonia 
on chest CT. Radiology. 2020; 296:E46-E54.

13. Qian K, Deng Y, Tai Y, Peng J, Peng H, Jiang L. Clinical characteristics of 2019 novel infected coronavirus pneumonia: a systemic review and meta-analysis. medRxiv. 2020 Jan 1

14. Shi H, Han X, Zheng C. Evolution of CT manifestations in a patient recovered from 2019 novel coronavirus (2019-nCoV) pneumonia in Wuhan, China. Radiology. 2020;295(1):20.
15. Chen N, Zhou M, Dong X, Qu J, Gong F, Han Y, et al. Epidemiological and clinical characteristics of 99 cases of 2019 novel coronavirus pneumonia in Wuhan, China: a descriptive study. Lancet. 2020 Feb 15;395(10223):507-13

16. Gao Y, Li T, Han M, Li X, Wu D, Xu Y, et al. Diagnostic utility of clinical laboratory data determinations for patients with the severe COVID-19. J Virol. J Med Virol. 2020;92:791796. 